\title{
Controllability of switched differential-algebraic equations
}

\author{
Ferdinand Küsters, Markus G.-M. Ruppert, Stephan Trenn \\ Fachbereich Mathematik, Technische Universität Kaiserslautern, Postfach 3049, 67653 Kaiserslautern, Germany
}

\begin{abstract}
We study controllability of switched differential algebraic equations. We are able to establish a controllability characterization where we assume that the switching signal is known. The characterization takes into account possible jumps induced by the switches. It turns out that controllability not only depends on the actual switching sequence but also on the duration between the switching times.
\end{abstract}

Keywords: switched systems, differential-algebraic systems; descriptor systems; controllability;

\section{Introduction}

In this paper we study controllability of switched differential-algebraic equations (switched DAEs) of the form

$$
E_{\sigma} \dot{x}=A_{\sigma} x+B_{\sigma} u,
$$

where $\sigma: \mathbb{R} \rightarrow \mathscr{P}$ for some parameter set $\mathscr{P}$ is a piecewise-constant switching signal (in particular, we assume that in each compact interval only finitely many switches occur). This class of systems plays an important role when modeling systems containing algebraic constraints together with sudden structural changes (e.g. electrical circuits), see [1]. We assume that the switching is given externally (e.g. induced by faults or coming from an external switching rule), i.e. $\sigma$ is given (and known) and not available for control. Hence here we view (1) as a special linear time-varying dynamical system and adapt the well established notion of controllability in the behavioral sense.

In general, controllability is a fundamental system property [2] concerned with the capability of influencing the system in a desired way through an appropriate choice of the control $u$. Controllability of time-varying linear DAEs has been studied for example in [3, 4, 5, 6], but all these approaches assume smooth coefficient matrices and are therefore not applicable to study switched DAEs. Controllability of switched ordinary differential equations (ODEs) is studied extensively in the literature, however most references view the switching signal Trenn)

Email address: trenn@mathematik.uni-kl.de (Stephan

Preprint submitted to Elsevier as a control input, see e.g. [7] and the references therein. There are surprisingly few references concerned with the notion of controllability for a given switching signal; apparently, the only exception is [8], which focuses on periodic switching signals. Hence a controllability characterization for an external switching signal will go beyond the state-of-the-art even for switched ODEs. Some preliminary results concerning the controllability of switched DAEs are presented in the conference contribution [9]; unfortunately, this publications contain some errors (in particular, the equivalence claimed in [9. Prop. 3.1] is not correct, c.f. Remark 3.7).

It should also be noted that [7] presents some duality between controllability and observability for switched linear ODEs (were the switching signal is seen as an input) and in the context of smoothly time-varying DAEs duality of observability and controllability was studied in [3]. It is not clear whether any reasonable duality notion also holds for linear switched DAEs (1); in particular, the connection between the controllability characterization presented here and the already known observability characterization for switched DAEs [10] is a topic of future research.

Our main contribution is the controllability characterization for switched DAEs given in Theorem 3.10 which heavily builds on the controllability characterization for the single-switch case in Theorem 3.6 Mathematical preliminaries like distributional solutions and controllability for non-switched DAEs are revisited in Section 2 .

We use the following notation: The real and natural numbers are denoted by $\mathbb{R}$ and $\mathbb{N}$, respectively. The indicator function for some interval $\mathscr{I} \subseteq \mathbb{R}$ is de-

January 22, 2015 
noted by $\mathbb{1}_{\mathscr{I}}$, i.e. $\mathbb{1}_{\mathscr{I}}(t)=1$ if $t \in \mathscr{I}$ and zero otherwise. The restriction of a function $f: \mathbb{R} \rightarrow \mathbb{R}$ to some interval $[a, b) \subseteq \mathbb{R}$ is $f_{[a, b)}:=\mathbb{1}_{[a, b)} f$; this restriction is also well defined for piecewise-smooth distributions (see Section 2.3). The space $\langle A, B\rangle \subseteq \mathbb{R}^{n}$ for matrices $A \in \mathbb{R}^{n \times n}, B \in \mathbb{R}^{n \times m}$ denotes the smallest $A$ invariant subspace which contains $\operatorname{im} B$, i.e. $\langle A, B\rangle=$ $\operatorname{im}\left[B, A B, A^{2} B, \cdots, A^{n-1} B\right]$.

\section{Mathematical preliminaries}

\subsection{Regular matrix pairs}

Here we restrict our attention to the non-switched DAE

$$
E \dot{x}=A x+B u
$$

for matrices $E, A \in \mathbb{R}^{n \times n}$ and $B \in \mathbb{R}^{n \times m}$ with smooth (i.e. arbitrarily often differentiable) solutions $(x, u)$. Note, that it is possible to define weaker notions of solutions; however, we will consider distributional solutions later on anyway which encompasses basically all other possible solution concepts. For existence and uniqueness of solutions regularity of the matrix pair $(E, A)$ is important:

Definition 2.1 (Regularity). A matrix pair $(E, A) \in$ $\mathbb{R}^{n \times n} \times \mathbb{R}^{n \times n}$ is called regular if, and only if, $\operatorname{det}(s E-$ $A$ ) is not the zero polynomial.

The following important well known characterizations for regularity hold (see e.g. [1, Thm. 6.3.2]).

Proposition 2.2 (Regularity characterizations). The following statements are equivalent:

(i) $(E, A)$ is regular.

(ii) There exist invertible $S, T \in \mathbb{R}^{n \times n}$ such that $(E, A)$ is transformed into a quasi-Weierstrass form (QWF):

$$
(S E T, S A T)=\left(\left[\begin{array}{cc}
I & 0 \\
0 & N
\end{array}\right],\left[\begin{array}{ll}
J & 0 \\
0 & I
\end{array}\right]\right)
$$

where $N$ is a nilpotent matrix.

(iii) The DAE Ex $=A x+B u$ has a solution for all smooth inputs $u$ and each corresponding solution is uniquely determined by the initial value $x(0)$.

The transformation matrices $S$ and $T$ for obtaining the QWF (3) can easily be calculated via the Wongsequences [11]:

$$
\begin{aligned}
\mathscr{V}_{0}:=\mathbb{R}^{n}, & \mathscr{V}_{i+1}:=A^{-1}\left(E \mathscr{V}_{i}\right), & i=0,1,2, \ldots, \\
\mathscr{W}_{0}:=\{0\}, & \mathscr{W}_{i+1}:=E^{-1}\left(A \mathscr{W}_{i}\right), & i=0,1,2, \ldots,
\end{aligned}
$$

where $M \mathscr{S}:=\{M x \mid x \in \mathscr{S}\}$ and $M^{-1} \mathscr{S}:=$ $\{x \mid M x \in \mathscr{S}\}$ denote the image and preimage, respectively, of $\mathscr{S}$ under the matrix $M$. It is easily seen that these sequences of subspaces converge after finitely many steps; denote these limits as

$$
\mathscr{V}_{(E, A)}^{*}:=\bigcap_{i \in \mathbb{N}} \mathscr{V}_{i} \quad \text { and } \quad \mathscr{W}_{(E, A)}^{*}:=\bigcup_{i \in \mathbb{N}} \mathscr{W}_{i} .
$$

Choosing full rank matrices $V$ and $W$ such that $\operatorname{im} V=$ $\mathscr{V}_{(E, A)}^{*}$ and $\mathrm{im} W=\mathscr{W}_{(E, A)}^{*}$, the QWF can be obtained via the transformation matrices [12]:

$$
T=[V, W] \quad \text { and } \quad S=[E V, A W]^{-1} .
$$

The following "projectors" play an important role in the analysis of switched DAEs and were first introduced in [13].

Definition 2.3 (Consistency, differential, impulse projectors). Consider the regular matrix pair $(E, A)$ with QWF (3).

- The consistency projector is

$$
\Pi_{(E, A)}:=T\left[\begin{array}{ll}
I & 0 \\
0 & 0
\end{array}\right] T^{-1} .
$$

- The differential projector is

$$
\Pi_{(E, A)}^{\mathrm{diff}}:=T\left[\begin{array}{ll}
I & 0 \\
0 & 0
\end{array}\right] S .
$$

- The impulse projector is

$$
\Pi_{(E, A)}^{\mathrm{imp}}:=T\left[\begin{array}{ll}
0 & 0 \\
0 & I
\end{array}\right] S .
$$

In all three cases the block structure corresponds to the block structure of the QWF. Furthermore let

$$
\begin{aligned}
A^{\text {diff }}:=\prod_{(E, A)}^{\text {diff }} A, & E^{\text {imp }}:=\prod_{(E, A)}^{\text {imp }} E, \\
B^{\text {diff }}:=\prod_{(E, A)}^{\text {diff }} B, & B^{\text {imp }}:=\prod_{(E, A)}^{\text {imp }} B .
\end{aligned}
$$

Note that only the consistency projector is a projector in the usual sense; the differential and impulse projectors are not idempotent. Furthermore, the definitions do not depend on the specific choice of the transformation matrices $S$ and $T$ as it has been shown for the consistency projector in [14, Section 4.2.2] and can be shown analogously for differential and impulsive projector.

An important feature for DAEs is the so called consistency space defined as follows: 
Definition 2.4 (Consistency space). Consider the DAE (2), then the consistency space is

$$
\left\{\begin{array}{l|l}
x_{0} \in \mathbb{R}^{n} & \begin{array}{l}
\exists \text { smooth solution } x \text { of } \\
E \dot{x}=A x \text { with } x(0)=x_{0}
\end{array}
\end{array}\right\}
$$

and the augmented consistency space is

$$
\left\{\begin{array}{l|l}
x_{0} \in \mathbb{R}^{n} & \begin{array}{l}
\exists \text { smooth solution }(x, u) \text { of } \\
E \dot{x}=A x+B u \text { with } x(0)=x_{0}
\end{array}
\end{array}\right\} .
$$

Proposition 2.5 ([15]). Consider the DAE [2] then the consistency space is $\mathscr{V}_{(E, A)}^{*}$ and the augmented consistency space is $\overline{\mathscr{V}_{(E, A, B)}^{*}}:=\mathscr{V}_{(E, A)}^{*} \oplus\left\langle E^{\mathrm{imp}}, B^{\mathrm{imp}}\right\rangle$.

\subsection{Controllability of nonswitched DAEs}

For DAEs (2) there are many different notions of controllability, see e.g. the survey [16], here we will only consider controllability in the behavioral sense (or equivalently R-controllability):

Definition 2.6 (Controllability). The DAE (2) is called controllable if, and only if, for all solutions $\left(x_{1}, u_{1}\right)$ and $\left(x_{2}, u_{2}\right)$ of (2) there exists a solution $\left(x_{12}, u_{12}\right)$ and $T>0$ such that

$$
\begin{aligned}
\left(x_{12}, u_{12}\right)_{(-\infty, 0)} & =\left(x_{1}, u_{1}\right)_{(-\infty, 0)}, \\
\left(x_{12}, u_{12}\right)_{(T, \infty)} & =\left(x_{2}, u_{2}\right)_{(T, \infty)} .
\end{aligned}
$$

The controllable subspace is

$\mathscr{C}_{(E, A, B)}:=\left\{x_{0} \in \mathbb{R}^{n} \mid \begin{array}{c}\exists T>0 \exists(x, u) \text { smooth solution } \\ \text { of } 2): x(0)=x_{0} \wedge x(T)=0\end{array}\right\}$.

It is well known (see e.g. [16, Lem. 2.3]) that the controllable space is equal to the reachable space, i.e.

$\mathscr{C}_{(E, A, B)}=\left\{\begin{array}{l|l}x_{0} \in \mathbb{R}^{n} & \begin{array}{c}\exists T>0 \exists(x, u) \text { smooth solution } \\ \text { of (2): } x(0)=0 \wedge x(T)=x_{0}\end{array}\end{array}\right\}$,

in particular, the controllable space is invariant with respect to solutions of (2), i.e. for any solution $(x, u)$ of (2) with $x(0) \in \mathscr{C}_{(E, A, B)}$ it follows that $x(t) \in \mathscr{C}_{(E, A, B)}$ for all $t \in \mathbb{R}$. The following controllability characterizations are also well known (for the specific formulation used here, see [15, Cor. 4.5]).

Proposition 2.7. Consider the regular DAE (2) and the notation from (5) and Definition 2.3.

(i) (2) is controllable if, and only if,

$$
\left\langle A^{\text {diff }}, B^{\text {diff }}\right\rangle=\mathscr{V}_{(E, A)}^{*} .
$$

(ii) The controllable space of (2) is given by

$$
\mathscr{C}_{(E, A, B)}=\left\langle A^{\text {diff }}, B^{\text {diff }}\right\rangle \oplus\left\langle E^{\text {imp }}, B^{\text {imp }}\right\rangle .
$$

In particular, the DAE is controllable if, and only if, the controllable space equals the augmented consistency space, i.e. $\mathscr{C}_{(E, A, B)}=\overline{\mathscr{V}_{(E, A, B)}^{*}}$. Furthermore, the characterization does not depend on the time $T$, hence we can replace " $\exists T>0$ " by " $\forall T>0$ " in the definition of the controllable space.

Note that from $A^{\text {diff }} \Pi^{\text {imp }}=0$ it follows that $\mathscr{C}_{(E, A, B)}$, $\mathscr{V}_{(E, A, B)}^{*}$ and $\overline{\mathscr{V}_{(E, A, B)}^{*}}$ are all $A^{\text {diff-invariant, in particular }}$ for all $t \in \mathbb{R}$

$$
\mathrm{e}^{A^{\mathrm{diff}} t} \mathscr{M}=\mathscr{M}, \quad \mathscr{M} \in\left\{\mathscr{C}_{(E, A, B)}, \mathscr{V}_{(E, A, B)}^{*}, \overline{\mathscr{V}_{(E, A, B)}^{*}}\right\} .
$$

For the analysis of controllability the following Kalman controllability decomposition (KCD) is useful, which utilizes the following subspace inclusions

$$
\begin{aligned}
&\left\langle A^{\text {diff }}, B^{\text {diff }}\right\rangle \subseteq \operatorname{im} \Pi_{(E, A)}^{\text {diff }}=\mathscr{V}_{(E, A)}^{*}, \\
&\left\langle E^{\mathrm{imp}}, B^{\mathrm{imp}}\right\rangle \subseteq \operatorname{im} \Pi_{(E, A)}^{\mathrm{imp}}=\mathscr{W}_{(E, A)}^{*} .
\end{aligned}
$$

Proposition 2.8 (KCD, [15, Cor. 4.6]). Consider a regular DAE (2) with the notation from (5) and Definition 2.3. Choose full column rank matrices $V_{1}, V_{2}, W_{1}, W_{2}$ as follows:

$$
\begin{aligned}
\operatorname{im} V_{1} & =\left\langle A^{\text {diff }}, B^{\text {diff }}\right\rangle, & & \operatorname{im} W_{1}=\left\langle E^{\text {imp }}, B^{\text {imp }}\right\rangle \\
\mathscr{V}_{(E, A)}^{*} & =\operatorname{im} V_{1} \oplus \operatorname{im} V_{2}, & \mathscr{W}_{(E, A)}^{*} & =\operatorname{im} W_{1} \oplus \operatorname{im} W_{2} .
\end{aligned}
$$

Then $T=\left[\left[V_{1}, W_{1}\right],\left[V_{2}, W_{2}\right]\right]$ and $S=$ $\left[\left[E V_{1}, A W_{1}\right],\left[E V_{2}, A W_{2}\right]\right]^{-1}$ are invertible and transform the DAE into a $K C D$ :

$$
(S E T, S A T, S B)=\left(\left[\begin{array}{cc}
E_{11} & E_{12} \\
0 & E_{22}
\end{array}\right],\left[\begin{array}{cc}
A_{11} & A_{12} \\
0 & A_{22}
\end{array}\right],\left[\begin{array}{c}
B_{1} \\
0
\end{array}\right]\right),
$$

where

$$
\left(E_{11}, A_{11}, B_{1}\right)=\left(\left[\begin{array}{cc}
I & 0 \\
0 & N
\end{array}\right],\left[\begin{array}{ll}
J & 0 \\
0 & I
\end{array}\right],\left[\begin{array}{c}
B_{J} \\
B_{N}
\end{array}\right]\right)
$$

with nilpotent $N$ and

$$
\mathscr{C}_{\left(E_{11}, A_{11}, B_{1}\right)}=\mathbb{R}^{\mathrm{rank}\left[V_{1}, W_{1}\right]}, \quad \mathscr{C}_{\left(E_{22}, A_{22}, 0\right)}=\{0\},
$$

i.e. the KCD decouples the DAE into a controllable and a non-controllable part. 


\subsection{Distributional solutions}

The switched DAE (1) usually will not have classical solutions, because each mode of the switched DAE given by the DAE $E_{i} \dot{x}=A_{i} x+B_{i} u$ might have different (augmented) consistency spaces which enforce jumps in the state-variable $x$. We therefore utilize the piecewisesmooth distributional solution framework as introduced in [14], i.e. $x$ and $u$ are vectors of piecewise-smooth distributions given by

$\mathbb{D}_{\mathrm{pw} \mathscr{C}^{\infty}}:=\left\{\begin{array}{l|l}D=f_{\mathbb{D}}+\sum_{t \in T} D_{t} & \begin{array}{l}f \in \mathscr{C}_{\mathrm{pw}}^{\infty}, T \subseteq \mathbb{R} \text { is } \\ \operatorname{discrete}, \forall t \in T: D_{t} \in \\ \operatorname{span}\left\{\delta_{t}, \delta_{t}^{\prime}, \delta_{t}^{\prime \prime}, \ldots\right\}\end{array}\end{array}\right\}$,

where $\mathscr{C}_{\mathrm{pw}}^{\infty}$ denotes the space of piecewise-smooth functions, $f_{\mathbb{D}}$ denotes the regular distribution induced by $f$ and $\delta_{t}$ denotes the Dirac impulse with support $\{t\}$. For a piecewise smooth distribution $D=f_{\mathbb{D}}+\sum_{t \in T} D_{t} \in$ $\mathbb{D}_{\mathrm{pw} \mathscr{C}}{ }^{\infty}$ three types of "evaluation at time $t$ " are defined: left sided evaluation $D\left(t^{-}\right):=f\left(t^{-}\right)$, right sided evaluation $D\left(t^{+}\right):=f\left(t^{+}\right)$and the impulsive part $D[t]:=D_{t}$ if $t \in T$ and $D[t]=0$ otherwise. It can be shown (see e.g. [17]) that the space $\mathbb{D}_{\text {pw } \mathscr{C}^{\infty}}$ can be equipped with a multiplication, in particular, the multiplication of a piecewise-constant function with a piecewise-smooth distribution is well defined and the switched DAE (1) can be interpreted as an equation within the space of piecewise-smooth distributions. Hence the following solution behavior (depending on $\sigma$ ) is well defined:

$$
\mathscr{B}_{\sigma}:=\left\{(x, u) \in \mathbb{D}_{\mathrm{pw} \mathscr{C}^{\infty}}^{n+m} \mid E_{\sigma} \dot{x}=A_{\sigma} x+B_{\sigma} u\right\},
$$

and restrictions of $x$ and $u$ to intervals are well defined as well. In this solution framework existence and uniqueness of solutions for (1) can be established:

Proposition 2.9 ([1, Cor. 6.5.2]). Consider the switched $D A E$ (1) with regular matrix pairs $\left(E_{p}, A_{p}\right)$ for all $p \in$ $\mathscr{P}$ and assume that the piecewise constant $\sigma$ is constant on $(-\infty, 0)$. Then for all inputs $u \in \mathbb{D}_{\mathrm{pw} \mathscr{C}^{\infty}}^{m}$ there exists $a$ solution $x \in \mathbb{D}_{\mathrm{pw} \mathscr{C}^{\infty}}^{n}$ of (1) and each solution is uniquely determined by $x\left(0^{-}\right) \in \overline{\mathscr{V}_{\sigma\left(0^{-}\right)}^{*}}$, where $\overline{\mathscr{V}_{\sigma\left(0^{-}\right)}^{*}}$ denotes the augmented consistency space of the active mode on $(-\infty, 0)$. Furthermore, for any consistent $x_{0} \in \overline{\mathscr{V}_{\sigma\left(0^{-}\right)}^{*}}$ there exists $(x, u) \in \mathfrak{B}_{\sigma}$ with $x\left(0^{-}\right)=x_{0}$.

In the following we will call (1) a regular switched $D A E$ if the assumptions of Proposition 2.9 are satisfied. Note that in particular the jumps and Dirac impulses induced by switches are uniquely determined in this solution framework (in contrast to other approaches, where for example additional glueing conditions are imposed, see e.g. [18]).
Remark 2.10. Let the feasibility space at $s^{ \pm}$of (1) be given by

$$
\mathscr{F}_{\sigma}^{s^{ \pm}}:=\left\{x\left(s^{ \pm}\right) \in \mathbb{R}^{n} \mid(x, u) \in \mathfrak{B}_{\sigma}\right\} .
$$

Clearly,

$$
\mathscr{F}_{\sigma}^{s^{ \pm}} \subseteq \overline{\mathscr{V}_{\sigma\left(s^{ \pm}\right)}^{*}}
$$

but equality does not hold in general. For example if in mode $\sigma\left(s^{-}\right)$the mode's equation is $0=x$ and for mode $\sigma\left(s^{+}\right)$the DAE is an ODE, then $\mathscr{F}_{\sigma}^{s^{+}}=\{0\}$ but $\overline{\mathscr{V}_{\sigma\left(s^{+}\right)}^{*}}=\mathscr{V}_{\sigma\left(s^{+}\right)}^{*}=\mathbb{R}^{n}$. However, if $\sigma$ is constant on $(-\infty, s)$ then $\mathscr{F}_{\sigma}^{s^{-}}=\overline{\mathscr{V}_{\sigma\left(s^{-}\right)}^{*}}$.

A side effect of the considered distributional solution framework is the possibility to consider impulsive controls, i.e. we allow for Dirac impulses in $u$. It is a well known fact, that for classical ODE systems any controllable states can be controlled to each other in arbitrarily short time; the following result shows that it is even possible to control two states instantaneously to each other when allowing impulsive controls:

Lemma 2.11 (Instantaneous control). Consider the (nonswitched) DAE (2) with corresponding controllable space $\mathscr{C}_{(E, A, B)}$. Then for all $x_{0}, x_{1} \in \mathscr{C}_{(E, A, B)}$ there exists $a$ (distributional) solution $(x, u)$ of (2) with $x\left(0^{-}\right)=x_{0}$ and $x\left(0^{+}\right)=x_{1}$.

Proof. Due to linearity it suffices to show that any controllable state $x_{1} \in \mathscr{C}_{(E, A, B)}$ is instantaneously reachable, i.e. there exists an input $u \in \mathbb{D}_{\mathrm{pw} \mathscr{C}^{\infty}}^{m}$ such that the corresponding solution $x \in \mathbb{D}_{\text {pw } \mathscr{C}^{\infty}}^{n}$ satisfies $x\left(0^{-}\right)=0$ and $x\left(0^{+}\right)=x_{1}$. Without restriction we can assume that the DAE is already in KCD as in Proposition 2.8, hence it remains to be shown that for any $v_{1} \in \mathbb{R}^{n_{1}}, w_{1} \in \mathbb{R}^{n_{2}}$, where $n_{1}:=\operatorname{dim}\left\langle A^{\text {diff }}, B^{\text {diff }}\right\rangle$ and $n_{2}:=\operatorname{dim}\left\langle E^{\text {imp }}, B^{\text {imp }}\right\rangle$, we can choose $u$ such that the solutions of

$$
\begin{aligned}
\dot{v} & =J v+B_{J} u, & v\left(0^{-}\right) & =0, \\
N \dot{w} & =w+B_{N} u, & w\left(0^{-}\right) & =0,
\end{aligned}
$$

satisfy $v\left(0^{+}\right)=v_{1}, w\left(0^{+}\right)=w_{1}$. First note that (see e.g. [1])

$$
w\left(0^{+}\right)=-\sum_{i=0}^{n_{2}-1} N^{i} B_{N} u^{(i)}\left(0^{+}\right) .
$$

Due the properties of the $\mathrm{KCD} \operatorname{dim}\left\langle N, B_{N}\right\rangle=n_{2}$ we can find $u^{0}, u^{1}, \ldots, u^{n_{2}-1} \in \mathbb{R}^{m}$ such that

$$
-\sum_{i=0}^{n_{2}-1} N^{i} B_{N} u^{i}=w_{1}
$$


Set $u$ on $(0, \varepsilon)$ (for some $\varepsilon>0$ ) equal to the polynomial $\sum_{i=0}^{n_{2}-1} \frac{t^{i}}{i !} u^{i}$ then we achieve $w\left(0^{+}\right)=w_{1}$ as required. To construct $u$ such that $v\left(0^{+}\right)=v_{1}$, we make the following ansatz for the impulsive parts of $u$ and $v$ at $t=0$ :

$$
u[0]=\sum_{i=0}^{n_{1}-1} \hat{u}^{i} \delta_{0}^{(i)} \text { and } \quad v[0]=\sum_{i=0}^{n_{1}-1} v^{i} \delta_{0}^{(i)}
$$

These impulsive parts must obey the differential equation

$$
\dot{v}[0]=J v[0]+B_{J} u[0],
$$

which can be rewritten as

$$
\begin{aligned}
v\left(0^{+}\right) & =J v^{0}+B_{J} \hat{u}^{0}, \\
v^{0} & =J v^{1}+B_{J} \hat{u}^{1}, \\
\vdots & \\
v^{n_{1}-2} & =J v^{n_{1}-1}+B_{J} \hat{u}^{n_{1}-1}, \\
v^{n_{1}-1} & =0 .
\end{aligned}
$$

Hence,

$$
v\left(0^{+}\right)=\sum_{i=0}^{n-1} J^{i} B_{J} \hat{u}^{i} .
$$

Due to the KCD, we have $\operatorname{dim}\left\langle J, B_{J}\right\rangle=n_{1}$ and we can always achieve $v\left(0^{+}\right)=v_{1}$ by choosing $u[0]$ accordingly (which can be done independently from the choice of $u$ on the interval $(0, \varepsilon))$.

Remark 2.12. The proof of Lemma 2.11 also shows that the controllable space $\mathscr{C}_{(E, A, B)}$ does not change when considering the regular DAE $E \dot{x}=A x+B u$ within the piecewise-smooth distributional solution framework, i.e.

$\mathscr{C}_{(E, A, B)}=\left\{\begin{array}{l|l}x_{0} \in \mathbb{R}^{n} & \begin{array}{l}\exists T \geq 0 \exists(x, u) \mathbb{D}_{\mathrm{pw} \mathscr{C}^{\infty} \text {-solution }} \\ \text { of }(2): x\left(0^{-}\right)=x_{0} \wedge x\left(T^{+}\right)=0\end{array}\end{array}\right\}$,

where $\subseteq$ follows trivially for $T>0$ and from Lemma 2.11 for $T=0$; while $\supseteq$ follows from the fact (concluded from the proof of Lemma 2.11) that additional jumps and Dirac impulses in $u$ can only produce jumps within the subspaces $\left\langle E^{\text {imp }}, B^{\text {imp }}\right\rangle$ and $\left\langle A^{\text {diff }}, B^{\text {diff }}\right\rangle$, respectively. Hence the smooth controllable space $\mathscr{C}_{(E, A, B)}=\left\langle A^{\text {diff }}, B^{\text {diff }}\right\rangle \oplus\left\langle E^{\text {imp }}, B^{\text {imp }}\right\rangle$ is not enlarged by allowing jumps and Dirac impulses in $u$.

\section{Controllability of switched DAEs}

\subsection{Controllability definition}

Definition 3.1 (Controllability). The switched DAE (1) is called controllable if, and only if, the corresponding solution behavior $\mathfrak{B}_{\sigma}$ is controllable in the behavioral sense on some interval $[0, T]$, i.e.

$$
\begin{aligned}
\forall\left(x_{1}, u_{1}\right),\left(x_{2}, u_{2}\right) \in & \mathfrak{B}_{\sigma} \exists\left(x_{12}, u_{12}\right) \in \mathfrak{B}_{\sigma}: \\
\left(x_{12}, u_{12}\right)_{(-\infty, 0)} & =\left(x_{1}, u_{1}\right)_{(-\infty, 0)}, \\
\left(x_{12}, u_{12}\right)_{(T, \infty)} & =\left(x_{2}, u_{2}\right)_{(T, \infty)} .
\end{aligned}
$$

For $0<s<t$, the $[s, t]$-controllable space is

$$
\mathscr{C}_{\sigma}^{[s, t]}:=\left\{\begin{array}{l|l}
x_{0} \in \mathbb{R}^{n} & \begin{array}{l}
\exists(x, u) \in \mathscr{B}_{\sigma}: \\
x\left(s^{-}\right)=x_{0} \wedge x\left(t^{+}\right)=0
\end{array}
\end{array}\right\} .
$$

Remarks 3.2. (i) It is easily seen (e.g. [9, Lemma 1.2]) that controllability as above is equivalent to zero-controllability, i.e.

$$
\begin{gathered}
\forall(x, u) \in \mathfrak{B}_{\sigma} \exists\left(x_{0}, u_{0}\right) \in \mathfrak{B}_{\sigma}: \\
\left(x_{0}, u_{0}\right)_{(-\infty, 0)}=(x, u)_{(-\infty, 0)} \wedge\left(x_{0}, u_{0}\right)_{(T, \infty)}=0 .
\end{gathered}
$$

This motivates the definition of the $[s, t]$ controllable space; in particular, we have the following controllability characterization:

$$
\mathscr{F}_{\sigma}^{0^{-}} \subseteq \mathscr{C}_{\sigma}^{[0, T]}
$$

Note that $\mathscr{F}_{\sigma}^{0^{-}} \supseteq \mathscr{C}_{\sigma}^{[0, T]}$ trivially holds.

(ii) Unlike the unswitched case the $[s, t]$-controllable space is usually not equal to the correspondingly defined $[s, t]$-reachable space (this difference between the controllable and reachable space is a well known property of general time-varying systems).

(iii) If a regular switched DAE (1) is considered the controllability definition can be reformulated as follows: For all consistent $x_{0}$ there exists an input $u$ defined on some interval $[0, T]$ and zero on $(T, \infty)$ such that the solution $x$ with initial condition $x\left(0^{-}\right)=x_{0}$ satisfies $x\left(T^{+}\right)=0$.

(iv) Combining Lemma 2.11 and Remark 2.12 we can conclude that for regular switched DAE the following property of the controllable space holds:

$$
\mathscr{C}_{\sigma}^{[s, t]}=\mathscr{C}_{\sigma}^{[s, t+\varepsilon]},
$$

where $\varepsilon>0$ is such that the interval $(t, t+\varepsilon]$ does not contain a switching time. In particular, any distributional input controlling $x_{0} \in \mathscr{C}_{\sigma}^{[s, t]}$ towards zero on the interval $[s, t]$ can be replaced by a classical control input achieving the same control action on the slightly larger interval $[s, t+\varepsilon]$. 
The following Lemma shows that we can assume that any control input can be chosen identically zero on $[0, \varepsilon)$ for sufficiently small $\varepsilon$ without changing the controllable space.

Lemma 3.3. Consider the regular switched DAE (1) and assume $(x, u) \in \mathfrak{B}_{\sigma}$ with $x\left(T^{+}\right)=0$ for some $T>$ 0 . Then there exists $(\widehat{x}, \widehat{u}) \in \mathfrak{B}_{\sigma}$ with $\widehat{x}\left(0^{-}\right)=x\left(0^{-}\right)$, $\widehat{x}\left(T^{+}\right)=0$ and $\widehat{u}_{[0, \varepsilon)}=0$ for some $\varepsilon>0$.

Proof. For simplicity we denote $(E, A, B):=$ $\left(E_{\sigma\left(0^{+}\right)}, A_{\sigma\left(0^{+}\right)}, B_{\sigma\left(0^{+}\right)}\right)$, then we have for sufficiently small $\varepsilon>0$, c.f. [1, Thm. 6.4.4]:

$$
x\left(\varepsilon^{+}\right)=e^{A^{\mathrm{diff}} \varepsilon} \Pi x\left(0^{-}\right)+x_{u}^{\varepsilon}-\sum_{i=0}^{n-1}\left(E^{\mathrm{imp}}\right)^{i} B^{\mathrm{imp}} u^{(i)}\left(\varepsilon^{+}\right),
$$

where

$$
x_{u}^{\varepsilon}:=\int_{0^{-}}^{\varepsilon^{+}} e^{A^{\mathrm{diff}}(t-\cdot)} B^{\mathrm{diff}} u \in\left\langle A^{\mathrm{diff}}, B^{\mathrm{diff}}\right\rangle
$$

is the only part of the solution depending on the input $u$ defined on $[0, \varepsilon]$. As shown in Lemma 2.11 it is possible to find $\widehat{u}[\varepsilon]$ such that 0 is controlled instantaneously to $x_{u}^{\varepsilon}$. This implies that together with $\widehat{u}_{[0, \varepsilon)}=0$, $\widehat{u}_{(\varepsilon, \infty)}=u_{(\varepsilon, \infty)}$ and linearity we have for the corresponding solution $\widehat{x}$ :

$$
\widehat{x}\left(\varepsilon^{+}\right)=x\left(\varepsilon^{+}\right) .
$$

Consequently, invoking uniqueness of solutions, $\widehat{x}_{(\varepsilon, \infty)}=x_{(\varepsilon, \infty)}$, in particular $\widehat{x}\left(T^{+}\right)=0$.

For convenience, let in the following $\mathscr{C}_{p}:=$ $\mathscr{C}_{\left(E_{p}, A_{p}, B_{p}\right)}$ and analogously for other matrices and spaces indexed by the corresponding matrix pair or triple.

We now would like to highlight the relationship between the two controllable spaces $\mathscr{C}_{\sigma}^{[s, t]}$ and $\mathscr{C}_{p}$.

Lemma 3.4. Consider the regular switched DAE (1) and assume that for $0 \leq s \leq t$ the switching signal $\sigma$ has no switches in the interval $[s, t]$, in particular $p:=\sigma\left(s^{-}\right)=\sigma\left(s^{+}\right)=\sigma\left(t^{-}\right)=\sigma\left(t^{+}\right)$. Then

$$
\mathscr{C}_{\sigma}^{[s, t]}=\mathscr{C}_{p} .
$$

Proof. First note that because of Remark 3.2[iv) it suffices to consider the case $s<t$.

If $x_{0} \in \mathscr{C}_{\sigma}^{[s, t]}$, then by definition there exists an input $u$ such that the corresponding solution $x$ of the switched DAE satisfies $x\left(s^{-}\right)=x_{0}$ and $x\left(t^{+}\right)=0$. In particular, $x_{0}$ is consistent for mode $p=\sigma\left(s^{-}\right)$and we can apply this input $u$ to the non-switched DAE $E_{p} \dot{x}=A_{p} x+B_{p} u$ with initial value $x\left(s^{-}\right)=x_{0}$. This yields exactly the same solution on the interval $[s, t+\varepsilon)$ for sufficiently small $\varepsilon>0$, in particular $x\left(t^{+}\right)=0$. Invoking Remark 2.12 together with time-invariance of the non-switched DAE we have $x_{0} \in \mathscr{C}_{\left(E_{p}, A_{p}, B_{p}\right)}$ and $\mathscr{C}_{\sigma}^{[s, t]} \subseteq \mathscr{C}_{\left(E_{p}, A_{p}, B_{p}\right)}$ is shown.

To show the converse, first observe that any smooth input controlling a feasible $x\left(s^{-}\right)=x_{0} \in \mathscr{C}_{p}$ towards $x\left(t^{+}\right)=0$ can be also applied to the switched systems with the same effect, i.e.

$$
\mathscr{C}_{p} \cap \mathscr{F}_{\sigma}^{s^{-}} \subseteq \mathscr{C}_{\sigma}^{[s, t]} .
$$

Hence it remains to be shown that any $x_{0} \in \mathscr{C}_{p}$ can actually be achieved by the switched system, i.e. $\mathscr{C}_{p} \subseteq \mathscr{F}_{\sigma}^{s^{-}}$. By assumption, $s$ is not a switching time, hence there exists $\varepsilon>0$ such that $\sigma$ is constantly $p$ also on $(s-\varepsilon, s)$. We can now choose a smooth input $u$ on $(s-\varepsilon, s)$ such that $x\left((s-\varepsilon)^{+}\right)=0$ is controlled to $x\left(s^{-}\right)=x_{0} \in \mathscr{C}_{p}$, which shows $\mathscr{C}_{p} \subseteq \mathscr{F}_{\sigma}^{s^{-}}$.

\subsection{The single switch case}

From a practical point of view, we have to assume that the control action starts at a certain point in time. Without restriction we therefore assume, that the input $u$ can only be chosen by us on the interval $[0, \infty)$. This does not exclude, that the input $u$ is non-zero on $(-\infty, 0)$, we just can't influence it in the past (in fact, this is already implied by Definition 3.1.

We will now consider the simplest nontrivial switched system where the switching signal has only one switch at the time $t_{s} \geq 0$ :

$$
\sigma^{\left\{t_{s}\right\}}(t):= \begin{cases}0, & t<t_{s} \\ 1, & t \geq t_{s}\end{cases}
$$

The controllability properties are very different for the two cases $t_{s}=0$ and $t_{s}>0$, because if $t_{s}=0$ then the input cannot take advantage of the controllability properties of the mode before the switch. In fact, any controllable switched system with switching time $t_{s}=0$ will also be controllable with switching time $t_{s}>0$ but not vice versa!

Example 3.5. Consider a switched DAE (1) with switching signal (7) and

$$
\begin{aligned}
& \left(E_{0}, A_{0}, B_{0}\right)=\left(\left[\begin{array}{ll}
1 & 0 \\
0 & 0
\end{array}\right],\left[\begin{array}{ll}
0 & 0 \\
0 & 1
\end{array}\right],\left[\begin{array}{l}
0 \\
1
\end{array}\right]\right), \\
& \left(E_{1}, A_{1}, B_{1}\right)=\left(\left[\begin{array}{ll}
0 & 0 \\
0 & 1
\end{array}\right],\left[\begin{array}{ll}
1 & 0 \\
0 & 0
\end{array}\right],\left[\begin{array}{l}
1 \\
0
\end{array}\right]\right) .
\end{aligned}
$$

For given input $u$, the solution of the switched DAE is given by

$$
x=\left(\begin{array}{l}
x_{1} \\
x_{2}
\end{array}\right)=\left(\begin{array}{c}
x_{0} \cdot \mathbb{1}_{\left(-\infty, t_{s}\right)}-u_{\left[t_{s}, \infty\right)} \\
-u_{\left(-\infty, t_{s}\right)}-u\left(t_{s}-\right) \cdot \mathbb{1}_{\left[t_{s}, \infty\right)}
\end{array}\right),
$$


for some $x_{0} \in \mathbb{R}$. If $t_{s}=0$ we cannot chose $u\left(t_{s}^{-}\right)$. If $u\left(0^{-}\right) \neq 0$ this means that $x_{2}(t) \neq 0$ for all $t>0$, i.e. the switched system is not controllable. If, however, $t_{s}>0$, then we are free to chose $u\left(t_{s}-\right)=0$ as well as $u_{\left[t_{s}, \infty\right)}=0$, hence $x_{\left[t_{s}, \infty\right)}=0$, which shows that now the switched system is controllable.

We are now ready to formulate our first main result:

Theorem 3.6 (Characterization of controllability: Single switch case). Consider the regular switched DAE (1) with switching signal (7). If $t_{s}=0$ then it holds

$$
\mathscr{C}_{\sigma\{0\}}^{[0, t]}=\Pi_{1}^{-1} \mathscr{C}_{1} \cap \overline{\mathscr{V}_{0}^{*}}
$$

for any $t>0$ and (1) is controllable if, and only if

$$
\Pi_{1}^{-1} \mathscr{C}_{1} \supseteq \overline{\mathscr{V}_{0}^{*}}
$$

If $t_{s}>0$ then it holds for $0 \leq s<t_{s}<t$

$$
\mathscr{C}_{\left.\sigma^{\{t s}\right\}}^{[s, t]}=\left(\mathscr{C}_{0}+e^{-A_{0}^{\mathrm{diff}}\left(t_{s}-s\right)} \Pi_{1}^{-1} \mathscr{C}_{1}\right) \cap \overline{\mathscr{V}_{0}^{*}}
$$

and (1) is controllable if, and only if,

$$
\mathscr{C}_{0}+\Pi_{1}^{-1} \mathscr{C}_{1} \supseteq \overline{\mathscr{V}_{0}^{*}}
$$

Proof. For $t_{s}=0$ we show (8).

" $\subseteq$ ": Let $x_{0} \in \mathscr{C}_{\sigma\{0\}}^{[0, t]} \subseteq \overline{\mathscr{V}_{0}^{*}}$, i.e. there exists an input $u$ with $(x, u) \in \mathfrak{B}_{\sigma\{0\}}$ and $x\left(0^{-}\right)=x_{0}, x\left(t^{+}\right)=0$. In particular, for all $\varepsilon \in(0, t)$ we have $x\left(\varepsilon^{-}\right) \in \mathscr{C}_{\sigma\{0\}}^{[\varepsilon, t]}=$ $\mathscr{C}_{1}$, where the latter follows from Lemma 3.4 . Hence $x\left(0^{+}\right)=\lim _{\varepsilon \searrow 0} x\left(\varepsilon^{-}\right) \in \mathscr{C}_{1}$. Without restriction, we can assume that $u[0]=0$ and $u^{(i)}\left(0^{+}\right)=0$ for all $i \in \mathbb{N}$ (cf. Lemma 3.3. Then (see e.g. [1, Thm. 6.5.1])

$$
x\left(0^{+}\right)=\Pi_{1} x\left(0^{-}\right),
$$

and therefore $x_{0}=x\left(0^{-}\right) \in \Pi_{1}^{-1} \mathscr{C}_{1}$, which shows $\mathscr{C}_{\sigma^{\{0\}}}^{[0, t]} \subseteq \Pi_{1}^{-1} \mathscr{C}_{1} \cap \overline{\mathscr{V}_{0}^{*}}$.

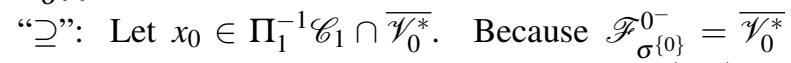
(c.f. Remark 2.10), there exists a solution $(x, u)$ of (1) with $x\left(0^{-}\right)=x_{0}$ and we may choose $u$ equal to zero on $[0, \varepsilon)$ for some $\varepsilon>0$. Then $x\left(0^{+}\right)=\Pi_{1} x_{0} \in$ $\Pi_{1}\left(\Pi_{1}^{-1} \mathscr{C}_{1} \cap \overline{\mathscr{V}_{0}^{*}}\right) \subseteq \Pi_{1} \Pi_{1}^{-1} \mathscr{C}_{1} \subseteq \mathscr{C}_{1}$ and consequently (by the $\mathrm{e}^{A_{1}^{\text {diff }}}$-invariance of $\left.\mathscr{C}_{1}\right) x\left(\varepsilon^{-}\right) \in \mathscr{C}_{1}$, hence there exists $u$ defined on $[\varepsilon, t]$ such that $x\left(t^{+}\right)=0$.

For $0 \leq s<t_{s}<t$ we show (9).

" $\subseteq$ ": From $s<t_{s}$ and $\sigma(s)=0$ it follows that

$$
\mathscr{C}_{0} \subseteq \mathscr{C}_{\sigma\{t s\}}^{[s, t]} \subseteq \overline{\mathscr{V}_{0}^{*}} \underset{2.5}{\stackrel{\text { Prop. }}{=}} \mathscr{V}_{0}^{*} \oplus\left\langle E_{0}^{\mathrm{imp}}, B_{0}^{\mathrm{imp}}\right\rangle
$$

From $\left\langle E_{0}^{\mathrm{imp}}, B_{0}^{\mathrm{imp}}\right\rangle \subseteq \mathscr{C}_{0} \subseteq \mathscr{C}_{\sigma^{\left\{t_{s}\right\}}}^{[s, t]}$ we can conclude

$$
\mathscr{C}_{\sigma}^{[s, t]}=\left(\mathscr{C}_{\left.\sigma^{\{t s}\right\}}^{[s, t]} \cap \mathscr{V}_{0}^{*}\right) \oplus\left\langle E_{0}^{\mathrm{imp}}, B_{0}^{\mathrm{imp}}\right\rangle
$$

Hence it suffices to show that

$$
\mathscr{C}_{\left.\sigma^{\{t s}\right\}}^{[s, t]} \cap \mathscr{V}_{0}^{*} \subseteq \mathscr{C}_{0}+e^{-A_{0}^{\mathrm{diff}}\left(t_{s}-s\right)} \Pi_{1}^{-1} \mathscr{C}_{1}
$$

Let $x_{0} \in \mathscr{C}_{\sigma^{\left\{t s_{s}\right\}}}^{[s, t]} \cap \mathscr{V}_{0}^{*} \subseteq \mathscr{F}_{\sigma^{\left\{t_{s}\right\}}}^{s^{-}}$then there exists $(x, u) \in$ $\mathfrak{B}_{\sigma^{\left\{t_{s}\right\}}}$ with $x\left(s^{-}\right)=x_{0}$ and $x\left(t^{+}\right)=0$. This implies $x\left(t_{s}^{+}\right) \in \mathscr{C}_{1}$. Due to Lemma 3.3 (applied to the interval $\left.\left[t_{s}, t\right]\right)$ we can assume that the input $u$ is zero on $\left[t_{s}, t_{s}+\varepsilon\right)$ for some $\varepsilon \in\left(0, t-t_{s}\right)$ and it follows that

$$
x\left(t_{s}^{-}\right) \in \Pi_{1}^{-1}\left\{x\left(t_{s}^{+}\right)\right\} \subseteq \Pi_{1}^{-1} \mathscr{C}_{1} .
$$

Since $x_{0} \in \mathscr{V}_{0}^{*}$ there exists $(\bar{x}, 0) \in \mathfrak{B}_{\left.\sigma^{\{t}\right\}}$ with $\bar{x}\left(s^{-}\right)=$ $x_{0}$. By linearity, $\hat{x}:=x-\bar{x}$ is a solution of (1) with initial value $\hat{x}\left(s^{-}\right)=0$ and input $u$. In particular, $\hat{x}\left(t_{s}^{-}\right)$is reachable (and hence controllable) for the unswitched DAE $E_{0} \dot{x}=A_{0} x+B_{0} u$, i.e.

$$
\hat{x}\left(t_{s}^{-}\right) \in \mathscr{C}_{0}
$$

This implies (invoking [13, Lem. 3])

$$
\mathrm{e}^{A_{0}^{\mathrm{diff}}\left(t_{s}-s\right)} x_{0}=\bar{x}\left(t_{s}^{-}\right)=x\left(t_{s}^{-}\right)-\hat{x}\left(t_{s}^{-}\right) \in \Pi_{1}^{-1} \mathscr{C}_{1}+\mathscr{C}_{0} .
$$

From the $\mathrm{e}^{A_{0}^{\mathrm{diff}}}$-invariance of $\mathscr{C}_{0}$ we can conclude

$$
x_{0} \in \mathscr{C}_{0}+\mathrm{e}^{-A_{0}^{\mathrm{diff}}\left(t_{s}-s\right)} \Pi_{1}^{-1} \mathscr{C}_{1}
$$

and 11) is shown.

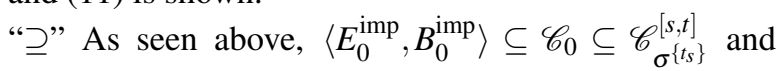
$\overline{\mathscr{V}_{0}^{*}}=\mathscr{V}_{0}^{*} \oplus\left\langle E_{0}^{\mathrm{imp}}, B_{0}^{\mathrm{imp}}\right\rangle$, hence it suffice to show

$$
\left(\mathrm{e}^{-A_{0}^{\mathrm{diff}}\left(t_{s}-s\right)} \Pi_{1}^{-1} \mathscr{C}_{1}+\mathscr{C}_{0}\right) \cap \mathscr{V}_{0}^{*} \subseteq \mathscr{C}_{\sigma^{\left\{t_{s}\right\}}}^{[s, t]}
$$

Let $x_{0} \in\left(\mathrm{e}^{-A_{0}^{\text {diff }}\left(t_{s}-s\right)} \Pi_{1}^{-1} \mathscr{C}_{1}+\mathscr{C}_{0}\right) \cap \mathscr{V}_{0}^{*}$, then there exists $(\bar{x}, 0) \in \mathfrak{B}_{\left.\sigma_{\{t}\right\}}$ with $\bar{x}\left(s^{-}\right)=x_{0}$ (because there is no switching on $(-\infty, s))$. This solution satisfies

$$
\begin{aligned}
\bar{x}\left(t_{s}^{-}\right) & =\mathrm{e}^{A_{0}^{\text {diff }}\left(t_{s}-s\right)} x_{0} \\
& \in \mathrm{e}^{A_{0}^{\text {diff }}\left(t_{s}-s\right)}\left(\mathrm{e}^{-A_{0}^{\mathrm{diff}}\left(t_{s}-s\right)} \Pi_{1}^{-1} \mathscr{C}_{1}+\mathscr{C}_{0}\right) \\
& =\Pi_{1}^{-1} \mathscr{C}_{1}+\mathscr{C}_{0}
\end{aligned}
$$

because $\mathscr{C}_{0}$ is $\mathrm{e}^{A_{0}^{\text {diff }}}$-invariant. We can choose $x_{1} \in \mathscr{C}_{0}$ such that

$$
\bar{x}\left(t_{s}^{-}\right)+x_{1} \in \Pi_{1}^{-1} \mathscr{C}_{1}
$$


Furthermore, we can choose an input $u$ defined on $\left[s, t_{s}\right)$ such that the corresponding solution $\hat{x}$ with $\hat{x}\left(s^{-}\right)=0$ fulfills $\hat{x}\left(t_{s}^{-}\right)=x_{1}$ as controllable and reachable space are equal for unswitched DAEs. By linearity, the solution $x=\bar{x}+\hat{x}$ of the switched DAE with initial condition $x\left(s^{-}\right)=x_{0}$ and input $u$ satisfies

$$
x\left(t_{s}^{-}\right)=\bar{x}\left(t_{s}^{-}\right)+x_{1} \in \Pi_{1}^{-1} \mathscr{C}_{1} .
$$

Choosing $u$ on $\left[t_{s}, t_{s}+\varepsilon\right)$ identically zero for some $\varepsilon \in$ $\left(0, t-t_{s}\right)$ we get

$$
x\left(t_{s}^{+}\right)=\Pi_{1} x\left(t_{s}^{-}\right) \in \mathscr{C}_{1}
$$

as well as $x\left(\left(t_{s}+\varepsilon\right)^{-}\right)=\mathrm{e}^{A_{1}^{\text {diff }}} \varepsilon x\left(t_{s}^{+}\right) \in \mathscr{C}_{1}$. Hence we can finally choose $u$ on $\left[t_{s}+\varepsilon, t\right]$ such that $x\left(t^{-}\right)=0$ and we have shown, that $x_{0} \in \mathscr{C}_{\sigma^{\left\{t_{s}\right\}}}^{[s, t]}$.

Controllability for $t_{s}=0$ and $t_{s}>0$.

By definition, c.f. (6), the switched DAE (1) with switching signal $\sigma^{\left\{t_{s}\right\}}$ is controllable if, and only if, for some $t>0$

$$
\mathscr{F}_{\sigma}^{0^{-}} \subseteq \mathscr{C}_{\sigma^{\left\{t_{s}\right\}}}^{[0, t]}
$$

Since $\sigma$ is constant on $(-\infty, 0)$ we have $\left.\mathscr{F}^{0} 0^{-} t_{s}\right\}=\overline{\mathscr{V}_{0}^{*}}$ for $t_{s}=0$ as well as $t_{s}>0$. This immediately shows the controllability characterization (8). For $t_{s}>0$, we can use (9) to show that (12) is equivalent to

$$
\overline{\mathscr{V}_{0}^{*}} \subseteq \mathscr{C}_{0}+\mathrm{e}^{-A_{0}^{\text {diff }}\left(t_{s}-s\right)} \Pi_{1}^{-1} \mathscr{C}_{1}
$$

and by invoking $\mathrm{e}^{A_{0}^{\mathrm{diff}}}$-invariance of $\mathscr{C}_{0}$ as well as $\overline{\mathscr{V}_{0}^{*}}$ we arrive at the desired controllability characterization.

The proof of Theorem 3.6 utilizes the fact that the subspace $\left\langle E_{0}^{\mathrm{imp}}, B_{0}^{\mathrm{imp}}\right\rangle$ is contained in the three subspaces $\mathscr{C}_{\sigma}^{[s, t]}, \mathscr{C}_{0}, \overline{\mathscr{V}_{0}^{*}}$ and the characterization seems independent of the actual size of $\left\langle E_{0}^{\mathrm{imp}}, B_{0}^{\mathrm{imp}}\right\rangle$ and one may wonder whether it is possible to characterize controllability independently of $\left\langle E_{0}^{\mathrm{imp}}, B_{0}^{\mathrm{imp}}\right\rangle$. Furthermore, the controllability characterization (Prop. 2.7) for unswitched DAEs is independent of $\left\langle E_{0}^{\mathrm{imp}}, B_{0}^{\mathrm{imp}}\right\rangle$. Unfortunately, this observation is misleading and actually lead to a partly wrong conclusion in [9], therefore, we would like to discuss this question in more detail with the following remark.

Remark 3.7. We can write $(10)$ also as

$$
\begin{aligned}
\left\langle A_{0}^{\text {diff }}, B_{0}^{\text {diff }}\right\rangle \oplus\left\langle E_{0}^{\text {imp }}, B_{0}^{\text {imp }}\right\rangle+ & \Pi_{1}^{-1}\left\langle A_{1}^{\text {diff }}, B_{1}^{\text {diff }}\right\rangle \\
& \supseteq \mathscr{V}_{0}^{*} \oplus\left\langle E_{0}^{\text {imp }}, B_{0}^{\text {imp }}\right\rangle,
\end{aligned}
$$

where we took into account that $\Pi_{1}^{-1}\left\langle E_{1}^{\mathrm{imp}}, B_{1}^{\mathrm{imp}}\right\rangle=$ $\Pi_{1}^{-1}\{0\}$. A further, simplification can be done by "substracting" $\left\langle E_{0}^{\mathrm{imp}}, B_{0}^{\mathrm{imp}}\right\rangle$ on both sides, resulting in the sufficient condition

$$
\left\langle A_{0}^{\text {diff }}, B_{0}^{\text {diff }}\right\rangle+\Pi_{1}^{-1}\left\langle A_{1}^{\text {diff }}, B_{1}^{\text {diff }}\right\rangle \supseteq \mathscr{V}_{0}^{*} .
$$

A necessary condition can be obtained from (10) by adding any complementing space of $\left\langle E_{0}^{\mathrm{imp}}, B_{0}^{\mathrm{imp}}\right\rangle$ within $\mathscr{W}_{0}^{*}$, resulting in the condition

$$
\mathscr{W}_{0}^{*} \oplus\left\langle A_{0}^{\text {diff }}, B_{0}^{\text {diff }}\right\rangle+\Pi_{1}^{-1}\left\langle A_{1}^{\text {diff }}, B_{1}^{\text {diff }}\right\rangle=\mathbb{R}^{n} .
$$

Despite their simple transformations the three conditions (10, 13) and (14) are not equivalent, in fact only the following holds

$$
\text { 13) } \Rightarrow \text { 10 }
$$

and we will show with the following two examples that the converse implications are not true.

Example $3.8([10) \nRightarrow(13))$. Consider the switched DAE (1) with switching signal (7) for $t_{s}>0$ and

$$
\begin{aligned}
& \left(E_{0}, A_{0}, B_{0}\right)=\left(\left[\begin{array}{ll}
1 & 0 \\
0 & 0
\end{array}\right],\left[\begin{array}{ll}
0 & 0 \\
0 & 0
\end{array}\right],\left[\begin{array}{l}
0 \\
1
\end{array}\right]\right), \\
& \left(E_{1}, A_{1}, B_{1}\right)=\left(\left[\begin{array}{ll}
1 & 0 \\
0 & 1
\end{array}\right],\left[\begin{array}{ll}
0 & 0 \\
0 & 0
\end{array}\right],\left[\begin{array}{l}
1 \\
1
\end{array}\right]\right) .
\end{aligned}
$$

Since the matrices are already in QWF, the following can simply be read off: $\mathscr{V}_{0}^{*}=\operatorname{im}\left[\begin{array}{l}1 \\ 0\end{array}\right], A_{0}^{\text {diff }}=0, B_{0}^{\text {diff }}=0$, $E_{0}^{\mathrm{imp}}=0, B_{0}^{\mathrm{imp}}=\left[\begin{array}{l}0 \\ 1\end{array}\right], \Pi_{1}=I, A_{1}^{\text {diff }}=0, B_{1}^{\text {diff }}=\left[\begin{array}{l}1 \\ 1\end{array}\right]$. Hence

$$
\mathscr{C}_{0}+\Pi_{1}^{-1} \mathscr{C}_{1}=\operatorname{im}\left[\begin{array}{l}
0 \\
1
\end{array}\right]+\operatorname{im}\left[\begin{array}{l}
1 \\
1
\end{array}\right]=\mathbb{R}^{2},
$$

i.e. (10) holds and the switched system is controllable. However, 13) is not satisfied:

$$
\begin{aligned}
\left\langle A_{0}^{\text {diff }}, B_{0}^{\text {diff }}\right\rangle+\Pi_{1}^{-1}\left\langle A_{1}^{\text {diff }}, B_{1}^{\text {diff }}\right\rangle= & \{0\}+\operatorname{im}\left[\begin{array}{l}
1 \\
1
\end{array}\right] \\
& \nsupseteq \operatorname{im}\left[\begin{array}{l}
1 \\
0
\end{array}\right]=\mathscr{V}_{0}^{*} .
\end{aligned}
$$

Example $3.9(\sqrt{14} \not \nRightarrow(10))$. Consider the switched DAE (1) with switching signal (7) for $t_{s}>0$ and

$$
\begin{aligned}
& \left(E_{0}, A_{0}, B_{0}\right)=\left(\left[\begin{array}{lll}
1 & 0 & 0 \\
0 & 0 & 0 \\
0 & 0 & 0
\end{array}\right],\left[\begin{array}{lll}
0 & 0 & 0 \\
0 & 0 & 0 \\
0 & 0 & 0
\end{array}\right],\left[\begin{array}{l}
0 \\
0 \\
1
\end{array}\right]\right), \\
& \left(E_{1}, A_{1}, B_{1}\right)=\left(\left[\begin{array}{lll}
1 & 0 & 0 \\
0 & 1 & 0 \\
0 & 0 & 1
\end{array}\right],\left[\begin{array}{lll}
0 & 0 & 0 \\
0 & 0 & 0 \\
0 & 0 & 0
\end{array}\right],\left[\begin{array}{l}
1 \\
1 \\
0
\end{array}\right]\right) \text {. }
\end{aligned}
$$

Since the matrices are already in QWF, we can easily read of the following: $\mathscr{V}_{0}^{*}=\operatorname{im}\left[\begin{array}{l}1 \\ 0 \\ 0\end{array}\right], \mathscr{W}_{0}^{*}=\operatorname{im}\left[\begin{array}{ll}0 & 0 \\ 1 & 0 \\ 0 & 1\end{array}\right]$, $A_{0}^{\text {diff }}=0, B_{0}^{\text {diff }}=0, E_{0}^{\text {imp }}=0, B_{0}^{\text {imp }}=\left[\begin{array}{l}0 \\ 0 \\ 1\end{array}\right], \Pi_{1}=I$, 


$$
\begin{aligned}
& A_{1}^{\text {diff }}=0, B_{1}^{\text {diff }}=\left[\begin{array}{l}
1 \\
1 \\
0
\end{array}\right] . \text { Hence } \\
& \qquad \mathscr{C}_{0}+\Pi_{1}^{-1} \mathscr{C}_{1}=\operatorname{im}\left[\begin{array}{l}
0 \\
0 \\
1
\end{array}\right]+\operatorname{im}\left[\begin{array}{l}
1 \\
1 \\
0
\end{array}\right] \\
& \qquad \nsupseteq \operatorname{im}\left[\begin{array}{l}
1 \\
0 \\
0
\end{array}\right]+\operatorname{im}\left[\begin{array}{l}
0 \\
0 \\
1
\end{array}\right]=\overline{\mathscr{V}_{0}^{*}},
\end{aligned}
$$

i.e. the switched system is not controllable. However, (14) is satisfied

$$
\begin{aligned}
\mathscr{W}_{0}^{*} \oplus\left\langle A_{0}^{\text {diff }}, B_{0}^{\text {diff }}\right\rangle & +\Pi_{1}^{-1}\left\langle A_{1}^{\text {diff }}, B_{1}^{\text {diff }}\right\rangle \\
& =\operatorname{im}\left[\begin{array}{ll}
0 & 0 \\
1 & 0 \\
0 & 1
\end{array}\right]+\{0\}+\operatorname{im}\left[\begin{array}{l}
1 \\
1 \\
0
\end{array}\right]=\mathbb{R}^{3} .
\end{aligned}
$$

\subsection{General switching signal}

We now consider an arbitrary piecewise constant switching signal $\sigma$ which is constant on $(-\infty, 0)$. Without restriction, we can relabel the matrices such that

$$
\sigma(t)= \begin{cases}-1, & t<t_{0}, \\ k, & t \in\left[t_{k}, t_{k+1}\right),\end{cases}
$$

where $0 \leq t_{0}<t_{1}<t_{2}<\ldots$ are the switching times of $\sigma$. Without restriction we can assume that $t_{0}=0$ (if there was no switch at $t=0$ we can artificially insert a switch to the same mode).

Our main result on controllability is the following.

Theorem 3.10. Consider the switched DAEs (1) with switching signal 15] and corresponding consistency projector $\Pi_{k}$, controllable space $\mathscr{C}_{k}$, augmented consistency space $\overline{\mathscr{V}_{k}^{*}}$ for each mode $k \in \mathbb{N} \cup\{-1\}$. For each $\ell \in \mathbb{N}$ define the following sequence of subspaces:

$$
\begin{aligned}
\mathscr{C}_{\ell}^{\ell} & :=\mathscr{C}_{\ell}, \\
\mathscr{C}_{k-1}^{\ell} & :=\mathscr{C}_{k-1}+e^{-A_{k-1}^{\mathrm{diff}}\left(t_{k}-t_{k-1}\right)} \Pi_{k}^{-1} \mathscr{C}_{k}^{\ell}, k=\ell, \ldots, 2,1 .
\end{aligned}
$$

Then (1) is controllable if, and only if, there exists $\ell \in \mathbb{N}$ such that

$$
\Pi_{0}^{-1} \mathscr{C}_{0}^{\ell} \supseteq \overline{\mathscr{V}_{-1}^{*}}
$$

Proof. We will first show, that for any $k, \ell \in \mathbb{N}$ with $0 \leq$ $k \leq \ell$

$$
\mathscr{C}_{k}^{\ell} \cap \overline{\mathscr{V}_{k}^{*}}=\mathscr{C}_{\sigma_{\geq t_{k}}}^{\left[t_{k}, t_{\ell}\right]}
$$

where

$$
\sigma_{\geq s}(t):= \begin{cases}\sigma\left(s^{+}\right), & t<s, \\ \sigma(t), & t \geq s\end{cases}
$$

i.e. we show that $\mathscr{C}_{k}^{\ell}$ (restricted to the corresponding augmented consistency space) equals all values at the $k$-th switching time which can be controlled towards zero on the interval $\left[t_{k}, t_{\ell}\right]$ under the assumption that the switching signal was constant in the past and at $t=t_{k}$.
We can use the identical proof of 9 where we replace $t_{s}$ by $t_{k}, s$ by $t_{k-1}, \mathscr{C}_{0}$ by $\mathscr{C}_{k-1}$ and $\mathscr{C}_{1}$ by $\mathscr{C}_{\sigma_{\geq t_{k}}}^{\left[t_{k}, t_{\ell}\right]}$ to obtain

$$
\begin{aligned}
& \mathscr{C}_{\sigma_{\geq t_{k-1}}}^{\left[t_{k-1}, t_{\ell}\right]} \\
& =\left(\mathscr{C}_{k-1}+e^{-A_{k-1}^{\mathrm{diff}}\left(t_{k}-t_{k-1}\right)} \Pi_{k}^{-1} \mathscr{C}_{\sigma_{\geq t_{k}}}^{\left[t_{k}, t_{\ell}\right]}\right) \cap \overline{\mathcal{V}_{k-1}^{*}} .
\end{aligned}
$$

Because $t_{\ell}$ is by definition not a switching instant of $\sigma_{\geq t_{\ell}}$ we have

$$
\mathscr{C}_{\sigma_{\geq t_{\ell}}}^{\left[t_{\ell}, t_{\ell}\right]} \stackrel{\underline{\underline{B .4}}}{\underline{\text { Lem. }}} \mathscr{C}_{\ell}=\mathscr{C}_{\ell} \cap \overline{\mathscr{V}_{\ell}^{*}}
$$

Hence, observing that for any matrix $M$ and subspace $\mathscr{S}, \mathscr{T}$ with $\operatorname{im} M \subseteq \mathscr{T}$ we have that

$$
M^{-1}(\mathscr{S} \cap \mathscr{T})=M^{-1} \mathscr{S}
$$

we inductively obtain

$$
\begin{aligned}
& \mathscr{C}_{\sigma_{\geq t_{k}}}^{\left[t_{k}, t_{\ell}\right]} \\
& \stackrel{[18]}{=}\left(\mathscr{C}_{k}+e^{-A_{k}^{\mathrm{diff}}\left(t_{k+1}-t_{k}\right)} \Pi_{k+1}^{-1} \mathscr{C}_{\sigma_{\geq t_{k+1}}\left[t_{t+1}, t_{\ell}\right]}\right) \cap \overline{\mathscr{V}_{k}^{*}} \\
& \stackrel{\text { Ind. }}{=}\left(\mathscr{C}_{k}+e^{-A_{k}^{\mathrm{diff}}\left(t_{k+1}-t_{k}\right)} \Pi_{k+1}^{-1}\left(\mathscr{C}_{k+1}^{\ell} \cap \overline{\mathscr{V}_{k+1}^{*}}\right)\right) \cap \overline{\mathscr{V}_{k}^{*}} \\
& \stackrel{[19]}{=}\left(\mathscr{C}_{k}+e^{-A_{k}^{\mathrm{diff}}\left(t_{k+1}-t_{k}\right)} \Pi_{k+1}^{-1} \mathscr{C}_{k+1}^{\ell}\right) \cap \overline{\mathscr{V}_{k}^{*}} \\
& =\mathscr{C}_{k}^{\ell} \cap \overline{\mathscr{V}_{k}^{*}},
\end{aligned}
$$

which shows (17).

Finally, we can use the same argument as in the " $t_{s}=$ 0 "-case of the proof of Theorem 3.6 to conclude that

$$
\mathscr{C}_{\sigma}^{\left[0, t_{\ell}\right]}=\left(\Pi_{0}^{-1} \mathscr{C}_{\sigma_{\geq 0}}^{\left[0, t_{\ell}\right]}\right) \cap \overline{\mathscr{V}_{-1}^{*}}=\left(\Pi_{0}^{-1} \mathscr{C}_{0}^{\ell}\right) \cap \overline{\mathscr{V}_{-1}^{*}}
$$

Hence, invoking $\overline{\mathscr{V}_{-1}^{*}}=\mathscr{F}_{\sigma}^{0^{-}}$, we have shown that the controllability characterization (6) is equivalent to 16 .

The dependencies of the switching times is crucial and in general cannot be avoided as the following example shows.

Example 3.11 (Dependency on the switching time). Consider the switched DAE (1) with switching signal (15) and

$$
\begin{aligned}
\left(E_{-1}, A_{-1}, B_{-1}\right) & =\left(\left[\begin{array}{ll}
1 & 0 \\
0 & 0
\end{array}\right],\left[\begin{array}{ll}
0 & 0 \\
0 & 1
\end{array}\right],\left[\begin{array}{l}
0 \\
0
\end{array}\right]\right), \\
\left(E_{0}, A_{0}, B_{0}\right) & =\left(\left[\begin{array}{ll}
1 & 0 \\
0 & 1
\end{array}\right],\left[\begin{array}{cc}
0 & 1 \\
-1 & 0
\end{array}\right],\left[\begin{array}{l}
0 \\
0
\end{array}\right]\right), \\
\left(E_{1}, A_{1}, B_{1}\right) & =\left(\left[\begin{array}{ll}
1 & 0 \\
0 & 1
\end{array}\right],\left[\begin{array}{ll}
0 & 0 \\
0 & 0
\end{array}\right],\left[\begin{array}{l}
0 \\
1
\end{array}\right]\right) \\
& =\left(E_{k}, A_{k}, B_{k}\right) \quad k \geq 2 .
\end{aligned}
$$


For any initial state $x_{0}=\left(x_{01}, x_{02}\right)^{T}$ the solution is given by

$$
\begin{aligned}
x(t)= & x_{0} \mathbb{1}_{(-\infty, 0)}+\left(\begin{array}{c}
\cos (t) x_{01} \\
-\sin (t) x_{01}
\end{array}\right) \mathbb{1}_{\left[0, t_{1}\right)} \\
& +\left(\begin{array}{c}
\cos \left(t_{1}\right) x_{01} \\
-\sin \left(t_{1}\right) x_{01}+\int_{t_{1}}^{t} u(s) \mathrm{d} s
\end{array}\right) \mathbb{1}_{\left[t_{1}, \infty\right)} .
\end{aligned}
$$

Thus the system is controllable, if, and only if, $\cos \left(t_{1}\right)=$ 0 , i.e. $t_{1}=\frac{\pi(1+2 k)}{2}, k \in \mathbb{N}$.

This result can also be obtained by applying Theorem 3.10 . Using $\mathscr{C}_{0}=\{0\}, \mathscr{C}_{1}=\operatorname{im}\left[\begin{array}{l}0 \\ 1\end{array}\right]$ and $\Pi_{0}=\Pi_{1}=$ $\left[\begin{array}{ll}1 & 0 \\ 0 & 1\end{array}\right]$ we get

$$
\mathscr{C}_{0}^{1}=\{0\}+\left[\begin{array}{cc}
\cos \left(t_{1}\right) & -\sin \left(t_{1}\right) \\
\sin \left(t_{1}\right) & \cos \left(t_{1}\right)
\end{array}\right] \operatorname{im}\left[\begin{array}{l}
0 \\
1
\end{array}\right]=\operatorname{im}\left[\begin{array}{c}
-\sin \left(t_{1}\right) \\
\cos \left(t_{1}\right)
\end{array}\right] .
$$

The condition (16) requires that the augmented consistency space $\overline{\mathscr{V}_{-1}^{*}}=\operatorname{im}\left[\begin{array}{l}1 \\ 0\end{array}\right]$ of the first mode is contained in $\Pi_{0}^{-1} \mathscr{C}_{0}^{1}=\mathscr{C}_{0}^{1}$. This is fulfilled iff $\cos \left(t_{1}\right)=0$, i.e $t_{1}=\frac{\pi(1+2 k)}{2}, k \in \mathbb{N}$.

Remark 3.12. For the single switch case with switching signal (7) and $t_{s}>0$ we have shown in Theorem 3.6 that controllability does not depend on the switching time $t_{s}>0$. In fact, for any switching signal given by (15) without switch at $t_{0}=0$ the controllability does not depend on $t_{1}>0$ (if the duration $t_{k+1}-t_{k}$ of each mode $k \in \mathbb{N}$ is fixed). The controllable space $\mathscr{C}_{\sigma}^{\left[0, t_{\ell}\right]}$ for any $\ell \geq 1$ is given by

$$
\begin{aligned}
\mathscr{C}_{\sigma}^{\left[0, t_{\ell}\right]}=\mathscr{C}_{\sigma_{\geq 0}}^{\left[0, t_{\ell}\right]} & =\mathscr{C}_{0}^{\ell} \cap \overline{\mathscr{V}_{0}^{*}} \\
& =\left(\mathscr{C}_{0}+\mathrm{e}^{-A_{0}^{\text {diff }}\left(t_{1}-0\right)} \Pi_{1}^{-1} \mathscr{C}_{1}^{\ell}\right) \cap \overline{\mathscr{V}_{0}^{*}},
\end{aligned}
$$

which equals $\overline{\mathscr{V}_{0}^{*}}$ if, and only if,

$$
\mathscr{C}_{0}+\Pi_{1}^{-1} \mathscr{C}_{1}^{\ell} \supseteq \overline{\mathscr{V}_{0}^{*}}
$$

because $\mathscr{C}_{0}$ and $\overline{\mathscr{V}_{0}^{*}}$ are $\mathrm{e}^{A_{0}^{\text {diff }}}$-invariant. This shows indeed that the controllability characterization does not depend on $t_{1}$. Nevertheless, this does not mean that the controllable spaces coincide for different $t_{1}>0$ if the system is not controllable.

\section{Conclusion}

We have presented a characterization of controllability for switched DAEs. This is the first result for controllability of switched DAEs and many important questions remain open. Duality and the connection between observability and controllability is a topic of ongoing research. Another question is whether the dependencies on the switching times can be relaxed, resulting in necessary or sufficient conditions for controllability. Finally, a long term goal is the construction of stabilizing controllers taking into account the special controllability features of switched DAEs.

\section{Acknowledgements}

We would like to thank the anonymous reviewers as well as Dr. Aneel Tanwani for their valuable and intriguing comments on the first version of this paper which lead to a significant improvement of the manuscript.

\section{References}

[1] S. Trenn, Switched differential algebraic equations, in: F. Vasca, L. Iannelli (Eds.), Dynamics and Control of Switched Electronic Systems - Advanced Perspectives for Modeling, Simulation and Control of Power Converters, Springer-Verlag, London, 2012, Ch. 6, pp. 189-216. doi : 10.1007/978-1-4471-2885-4_6

[2] R. E. Kalman, Mathematical description of linear dynamical systems, SIAM J. Control Optim. 1 (1963) 152-192. doi: 10.1137/0301010

[3] S. L. Campbell, N. K. Nichols, W. J. Terrell, Duality, observability, and controllability for linear time-varying descriptor systems, Circuits Systems Signal Process. 10 (4) (1991) 455-470. doi:10.1007/BF01194883

[4] C.-J. Wang, Controllability and observability of linear timevarying singular systems, IEEE Trans. Autom. Control 44 (10) (1999) 1901-1905. doi:10.1109/9.793733

[5] A. Ilchmann, V. Mehrmann, A behavioural approach to timevarying linear systems, Part 1: General theory, SIAM J. Control Optim. 44 (5) (2005) 1725-1747. doi:10.1137/ S0363012904442239

[6] A. Ilchmann, V. Mehrmann, A behavioural approach to timevarying linear systems, Part 2: Descriptor systems, SIAM J. Control Optim. 44 (5) (2005) 1748-1765. doi:10.1137/ 040609021

[7] Z. Sun, S. S. Ge, Switched linear systems, Communications and Control Engineering, Springer-Verlag, London, 2005. doi : 10 . 1007/1-84628-131-8

[8] J. Ezzine, A. Haddad, Controllability and observability of hybrid systems, Int. J. Control 49 (6) (1989) 2045 - 2055. doi:10. 1080/00207178908559761

[9] M. G.-M. Ruppert, S. Trenn, Controllability of switched DAEs: The single switch case, in: PAMM - Proc. Appl. Math. Mech., Vol. 14, Wiley-VCH Verlag GmbH, 2014, pp. 15-18. doi : 10. 1002/pamm. 201410005

[10] A. Tanwani, S. Trenn, Observability of switched differentialalgebraic equations for general switching signals, in: Proc. 51st IEEE Conf. Decis. Control, Maui, USA, 2012, pp. 26482653. doi:10.1109/CDC. 2012.6427087

[11] K.-T. Wong, The eigenvalue problem $\lambda T x+S x$, J. Diff. Eqns. 16 (1974) 270-280. doi:10.1016/0022-0396(74)90014-X

[12] T. Berger, A. Ilchmann, S. Trenn, The quasi-Weierstraß form for regular matrix pencils, Lin. Alg. Appl. 436 (10) (2012) 40524069. doi:10.1016/j.1aa.2009.12.036

[13] A. Tanwani, S. Trenn, On observability of switched differentialalgebraic equations, in: Proc. 49th IEEE Conf. Decis. Control, Atlanta, USA, 2010, pp. 5656-5661. doi:10.1109/CDC. 2010.5717685 
[14] S. Trenn, Distributional differential algebraic equations Ph.D. thesis, Institut für Mathematik, Technische Universität Ilmenau, Universitätsverlag Ilmenau, Ilmenau, Germany (2009).

URL http://www.db-thueringen.de/servlets/ DocumentServlet?id=13581

[15] T. Berger, S. Trenn, Kalman controllability decompositions for differential-algebraic systems, Syst. Control Lett. 71 (2014) 5461. doi:10.1016/j.sysconle.2014.06.004

[16] T. Berger, T. Reis, Controllability of linear differential-algebraic systems - a survey, in: A. Ilchmann, T. Reis (Eds.), Surveys in Differential-Algebraic Equations I, Differential-Algebraic
Equations Forum, Springer-Verlag, Berlin-Heidelberg, 2013, pp. 1-61. doi : 10.1007/978-3-642-34928-7_1

[17] S. Trenn, Regularity of distributional differential algebraic equations, Math. Control Signals Syst. 21 (3) (2009) 229-264. doi : 10.1007/s00498-009-0045-4

[18] P. Rocha, J. C. Willems, P. Rapisarda, D. Napp, On the stability of switched behavioral systems, in: Proc. 50th IEEE Conf. Decis. Control and European Control Conference ECC 2011, Orlando, USA, 2011, pp. 1534-1538. doi:10.1109/CDC. 2011. 6160860 